\title{
Concurrent multiple left atrial focal activations with fibrillatory conduction and right atrial focal or reentrant activation as the mechanism in atrial fibrillation
}

Takashi Nitta, MD

Yosuke Ishii, MD

Yasuo Miyagi, MD

Hiroya Ohmori, MD

Shun-ichiro Sakamoto, MD

Shigeo Tanaka, MD

See related editorial on page 627.
From the Nippon Medical School, Tokyo, Japan.

Presented at the Seventy-second and Seventy-third Scientific Sessions of The American Heart Association (Nov 7-10, 1999, in Atlanta, Ga, and Nov 12-15, 2000, in New Orleans, La).

Received for publication Jan 20, 2003; revisions requested March 24, 2003; revisions received April 6, 2003; accepted for publication May 14, 2003.

Address for reprints: Takashi Nitta, MD, Associate Professor, Cardiothoracic Surgery, Nippon Medical School, 1-1-5, Sendagi, Bunkyo-ku, Tokyo 113-8603, Japan (E-mail: nitta@nms.ac.jp).

J Thorac Cardiovasc Surg 2004;127:770-8 $0022-5223 / \$ 30.00$

Copyright ( $\odot 2004$ by The American Association for Thoracic Surgery

doi:10.1016/j.jtcvs.2003.05.001
Objective: We examined the atrial activation during atrial fibrillation to validate the rationale behind simplified surgical procedures.

Methods: Intraoperative mapping of the entire atrial epicardium was performed in 21 patients with permanent atrial fibrillation and mitral valve disease using a 256-channel, 3-dimensional dynamic mapping system.

Results: Concurrent multiple repetitive activations arose from the posterior left atrium adjacent to the pulmonary veins or the left atrial appendage in all patients. The fastest activation propagated toward the right atrium conducting through Bachmann's bundle, leaving the other activations confined to a small atrial region. As the activation propagated toward the right atrium, there was a progressive conduction delay or block in the pathway. As a result, the activation in the right atrium desynchronized with the left atrial activation and became irregular and complex. The average cycle length measured at the right atrial appendage was significantly longer than that at the left atrial foci (206 \pm 32 milliseconds vs $175 \pm$ 23 milliseconds, $P<.001$ ). In addition to the passive activation, a focal activation and reentrant activation were also observed in the right atrium in 5 and 6 patients, respectively. The number of wave fronts in the right atrium was significantly greater than that in the left atrium $(2.9 \pm 0.8$ vs $0.6 \pm 0.7, P<.001)$.

Conclusions: Multiple left atrial focal activations with fibrillatory conduction and right atrial focal or reentrant activations are the mechanism in permanent atrial fibrillation associated with mitral valve disease. Intraoperative mapping would facilitate the indication for simplified procedures confined to the left atrium or the pulmonary veins.

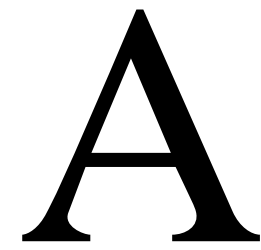

trial fibrillation (AF) is the most common supraventricular tachyarrhythmia carrying a significant morbidity and mortality. It is generally accepted that coexisting multiple wavelets ${ }^{1}$ are the underlying electrophysiology occurring in permanent AF. Multiple reentrant activations ${ }^{2}$ and a single reentrant activation of a very short cycle length with fibrillatory conduction ${ }^{3}$ have been shown to cause multiple wavelets in experimental studies. There have been a few mapping studies on atrial activation during AF in patients with or without structural heart disease. Macroreentrant activation or atrial reentry was demonstrated during electrically induced AF in patients with Wolf-Parkinson-White syndrome. ${ }^{4,5}$ Recent studies on permanent AF in patients with mitral valve disease have suggested that repetitive activation arising from the left atrium plays an important role in the 
perpetuation of $\mathrm{AF}$ in this subset of patients. ${ }^{6,7}$ Ablative procedures confined to the left atrium or the pulmonary veins have been shown to cure $\mathrm{AF}$ in selected patients. ${ }^{8,9}$ However, it has not been determined yet which mechanisms participate in the perpetuation of permanent AF in humans and the rationale behind the simplified ablative procedures has not been directly validated.

In the present study, we sought to characterize the atrial activation during permanent $\mathrm{AF}$ in patients with mitral valve disease. Analysis of the atrial activation using the usual static and 2-dimensional map is sometimes intricate because of coexisting multiple wavelets during AF and a 3-dimensional and complex structure of the atria. We used custom-made electrode patches with 253 electrodes and simultaneously mapped the entire atrial epicardium, including the posterior left atrium between the pulmonary veins. The maps were displayed in a dynamic mode as a movie on 3-dimensionally constructed atrial models to keep the atrial activation correlated with the anatomical landmarks, such as the atrioventricular annuli, great vessels, and the pulmonary vein orifices. These technical advantages allowed precise and accurate analysis of concurrent multiple wavelets in the atria.

\section{Methods \\ Patients}

The study included 21 patients with permanent AF who underwent surgery for AF and mitral valve disease. The study protocol was approved by the research committee of our institute and a written informed consent for intraoperative electrophysiological study was obtained from each patient. There were 7 male and 14 female patients with an average age of $60 \pm 10$ years old. The duration of AF was 3 years or more in all patients. Fourteen patients had rheumatic mitral valve stenosis and 7 patients had non-rheumatic mitral valve regurgitation. There were 8 patients who had moderate to severe tricuspid valve regurgitation and 5 patients with aortic valve regurgitation or stenosis. The left atrial dimension and left ventricular ejection fraction determined by transthoracic echocardiography were $53 \pm 11 \mathrm{~mm}$ and $52 \pm 13 \%$, respectively.

\section{Intraoperative Mapping}

Intraoperative mapping was performed before the patient was cannulated for cardiopulmonary bypass. Electrograms were recorded from the entire right and left atrial epicardial surface along with the electrocardiogram during AF. Following the data acquisition, the patients underwent the radial procedure ${ }^{10}$ for $\mathrm{AF}$, mitral valve surgery, and additional procedures to correct other structural heart diseases. The mapping electrode patches (Figure 1) were custom-made to fit the patches snugly to the entire atrial epicardium. A mold of a cadaver heart was made, and the curvature of the atrial epicardium was copied to 3 silicone sheets: the right atrial free wall, left atrial free wall including the posterior left atrium between the pulmonary veins, and the anterior atrium behind the great arteries. A total of 253 unipolar electrodes were distributed over the sheets with interelectrode distances of 5 to 7 $\mathrm{mm}$.

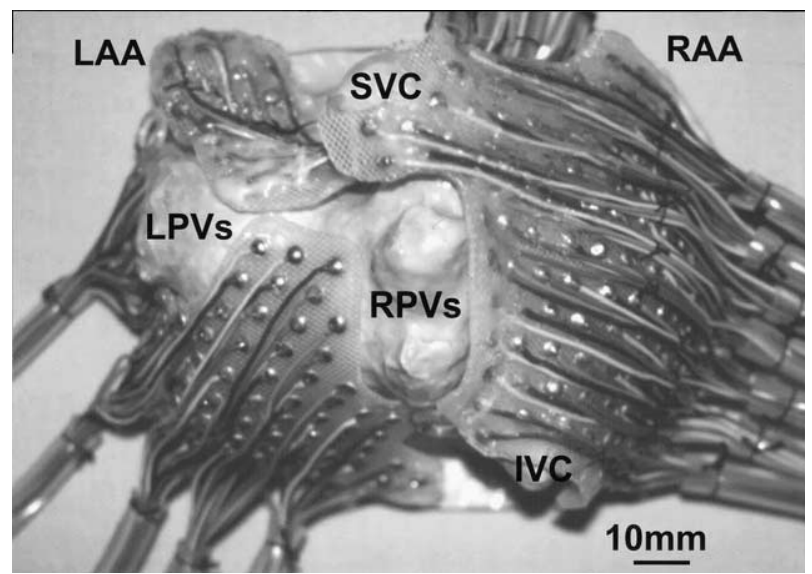

Figure 1. Electrode patches placed on a mold made from a cadaver heart. A total of 253 unipolar electrodes were distributed over the silicone patches that covered the entire epicardium of the right and left atria. $R A A$, Right atrial appendage; $S V C$, superior vena cava; $I V C$, inferior vena cava; $R P V s$, right pulmonary veins; $L P V s$, left pulmonary veins; $L A A$, left atrial appendage.

\section{Data Analysis}

A 256-channel dynamic mapping system was used for data acquisition and analysis. Data processing was performed on Silicon Graphics graphic workstations (Indigo2 and O2, Silicon Graphics Inc, Mountain View, Calif). Local activation times were determined as the maximum negative derivative of the unipolar electrogram. The minimal activation cycle length was set as 100 milliseconds to handle multiple deflections that occurred in regions of conduction block. Computer-picked activation times were verified manually. Activation maps were displayed in a dynamic mode as a movie with 3-dimensionally constructed atrial models on a computer. The 3-dimensional atrial models were constructed from the scanned images of cross sections of the cadaver atria.

A 4000-millisecond duration of data was analyzed to characterize the activation pattern in each patient. Reentry was defined as reactivation of a site by a wave front that continued from the preceding activation of the site. Focal activation was defined as an early activation without any late activation from a previous cycle adjacent to the early activation site. When an atrium was activated by a wave front that originated from the other atrium, the activation pattern was considered to be passive. The number of wave fronts was determined in each atrium over a 10-millisecond interval every 100 milliseconds. Continuous values were expressed as mean \pm 1 SD. Student $t$ test was used to compare data between 2 groups when appropriate.

\section{Results}

\section{Left Atrial Activation}

In all patients, concurrent multiple focal activations were demonstrated in the left atrium. Macroreentry circulating around an anatomical obstacle or atrial reentry was not observed in any patients. Multiple focal activations with a different cycle length arose from multiple left atrial sites at 

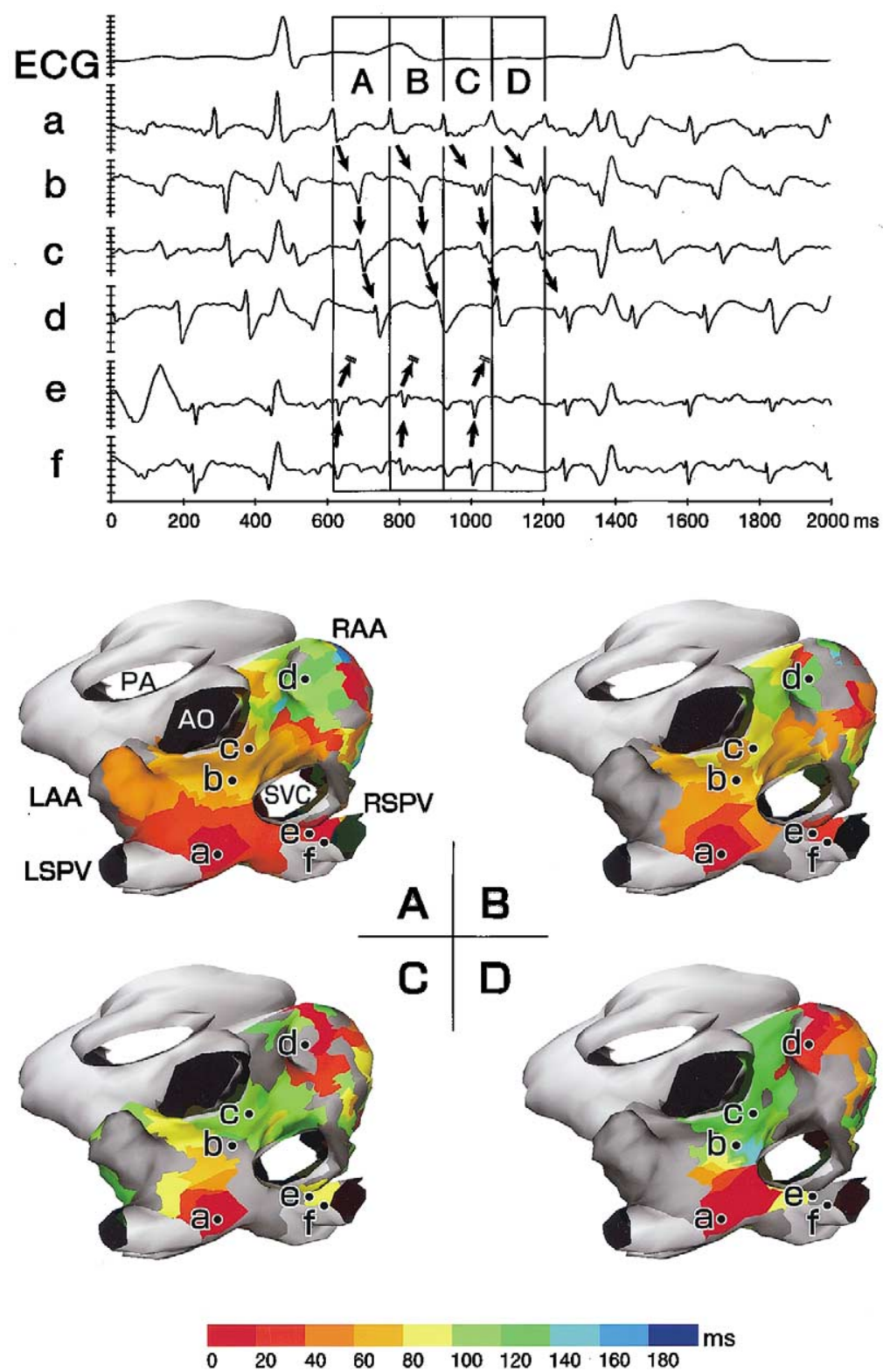

Figure 2. Atrial activation maps constructed in a 55-year-old male patient with permanent AF associated with aortic and mitral valve disease. The maps are shown along with an electrocardiogram and selected electrograms. The maps represent a superior view of the atria, as if being observed cranially. The location of the selected electrodes $(a-f)$ is indicated in the maps. The activation maps during the time windows from $A$ to $D$ are shown. The duration of each time window (the cycle length of the activation recorded from electrode a) is 164,144 , 140, and 141 milliseconds. The atrial activation is shown with color coding at 20 -millisecond increments. The gray regions represent regions where no activation is seen during the time window. See the text for an explanation. ECG, Electrocardiogram; $A O$, aorta; $P A$, pulmonary artery; $R A A$, right atrial appendage; $L A A$, left atrial appendage; $S V C$, superior vena cava; $R S P V$, right superior pulmonary vein; $L S P V$, left superior pulmonary vein.

the same time. The activation with the shortest cycle length propagated to the right atrium, leaving the other activations confined to a small atrial region. Figure 2 demonstrates the activation maps during AF constructed in a patient, which represent repetitive activations arising from 2 different left atrial regions adjacent to the right and left superior pulmonary veins. The activation arising from the left superior pulmonary vein (site a) was rapid and relatively regular, and the cycle length ranged from 132 to 160 milliseconds. In the meantime, the activation arising from the right superior 


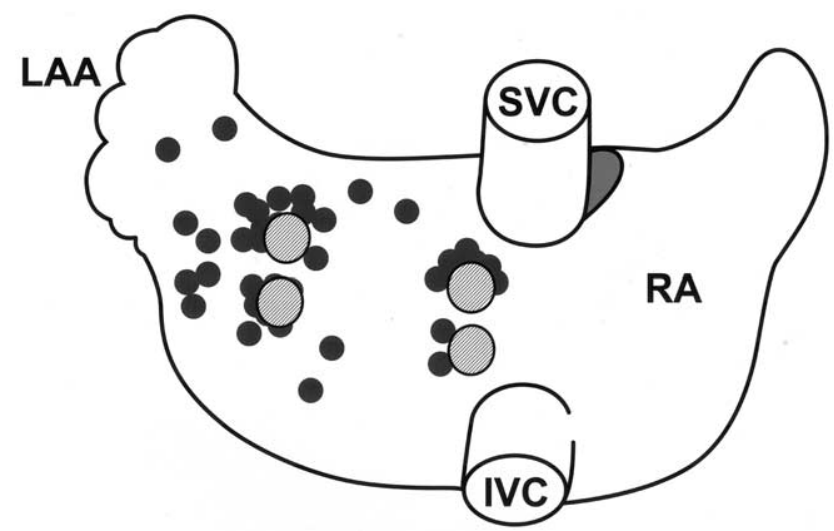

Figure 3. Distribution of focal activation in the left atrium. The schema represents the posterior aspect of the atria. The 4 shaded circles indicate the pulmonary vein orifices. The electrode locations where the focal activation arose are plotted on the schema as closed circles; $R A$, Right atrium.

pulmonary vein (site f) was slower and irregular, and the cycle length varied from 185 to 257 milliseconds. Because the activation arising from the left superior pulmonary vein was faster than that from the right superior pulmonary vein, only the activation from the left superior pulmonary vein conducted to Bachmann's bundle and the activation arising from the right superior pulmonary vein was confined to a small left atrial region around the vein.

Left-to-right interatrial conduction via Bachmann's bundle was demonstrated in all patients. There was a progressive conduction delay or block in the pathway from the focus to Bachmann's bundle and to the right atrium, resulting in a variable conduction ratio and nonactivated regions as shown in Figure 2. These characteristics of the left atrial activation and the interatrial conduction were demonstrated also in other patients.

All the patients had 2 to 4 foci (median 2) in the left atrium. A total of 50 foci were determined in 21 patients. The location of the focal activation in the left atrium is depicted in Figure 3. There was no difference in the location or cycle length of the foci between the rheumatic and nonrheumatic patients. The majority of the focal activations arose from the posterior left atrium adjacent to the pulmonary veins or the left atrial appendage. The cycle length was regular in some patients but varied and changed beat-bybeat in others. The cycle length of the repetitive activation ranged from 147 to 268 milliseconds (175 \pm 23 milliseconds). The average and the range of the cycle length of the focal activation arising from each left atrial site are summarized in Table 1. The cycle length of the activation arising from the left superior pulmonary vein was significantly shorter than that from the lateral left atrium.
TABLE 1. Cycle length of the focal activation

\begin{tabular}{lccc}
\hline & & \multicolumn{2}{c}{ Cycle length } \\
\cline { 3 - 4 } LA sites & $\mathbf{n}$ & Average (ms) & Range (ms) \\
\hline LSPV & 18 & $177 \pm 22^{*}$ & $230-147$ \\
RSPV & 10 & $183 \pm 33$ & $268-150$ \\
LIPV & 5 & $189 \pm 13$ & $210-179$ \\
RIPV & 2 & NA & $202-186$ \\
LAA & 5 & $187 \pm 15$ & $213-174$ \\
Lateral LA & 6 & $204 \pm 20^{*}$ & $236-186$ \\
Anterior LA & 2 & NA & $210-195$ \\
Posterior LA & 2 & NA & $204-186$
\end{tabular}

$L A$, Left atrium; $L S P V$, left superior pulmonary vein; $R S P V$, right superior pulmonary vein; $L I P V$, left inferior pulmonary vein; $R I P V$, right inferior pulmonary vein; $L A A$, left atrial appendage; $N A$, not applicable because of the small sample size.

${ }^{*}$ LSPV versus lateral LA, $P<.05$.

\section{Right Atrial Activation}

All patients showed a passive pattern of activation conducting from the left atrium via Bachmann's bundle as shown in Figure 2. As the activation propagated toward the right atrium, there was a progressive conduction delay or block in the pathway. The average cycle length measured at the right atrial appendage was significantly longer (206 \pm 32 milliseconds, $P<.001)$ than that at the left atrial foci. Progressive slowing of the conduction was also observed in the lateral right atrium as shown in Figure 4. The delayed conduction resulted in residual activation from the preceding cycle and coexisting multiple wavelets in the lateral right atrium. As a result, the activation in the right atrium desynchronized with the left atrial activation and became irregular and complex. The number of wave fronts in the right atrium was 2 to $4(2.9 \pm 0.8)$, which was significantly larger than that in the left atrium $(0.6 \pm 0.7, P<.001)$.

In addition to the passive activation pattern, atrial reentry (Figure 5) and focal activation (Figure 6) were also observed in 6 and 5 patients, respectively. In 2 patients, the pattern of activation was mixed. There was no correlation between the atrial activation patterns and the duration of $\mathrm{AF}$, right atrial pressure, or the presence or severity of tricuspid valve regurgitation. Figure 5 represents the right atrial reentrant activation seen in a patient. There were at least 2 reentrant circuits in the lateral right atrium: 1 was in the middle of the lateral right atrium and the other in the right atrial appendage. These reentrant circuits changed their location and configuration cycle-by-cycle. The pattern of left atrial activation was focal in this patient.

Figure 6 represents the focal pattern of right atrial activation. The site of the focus changed cycle-by-cycle. The activation arose from the middle of the lateral right atrium in the initial 2 consecutive cycles (left and middle maps in Figure 6), followed by focal activation from the crista terminalis and passive activation propagating from the left 

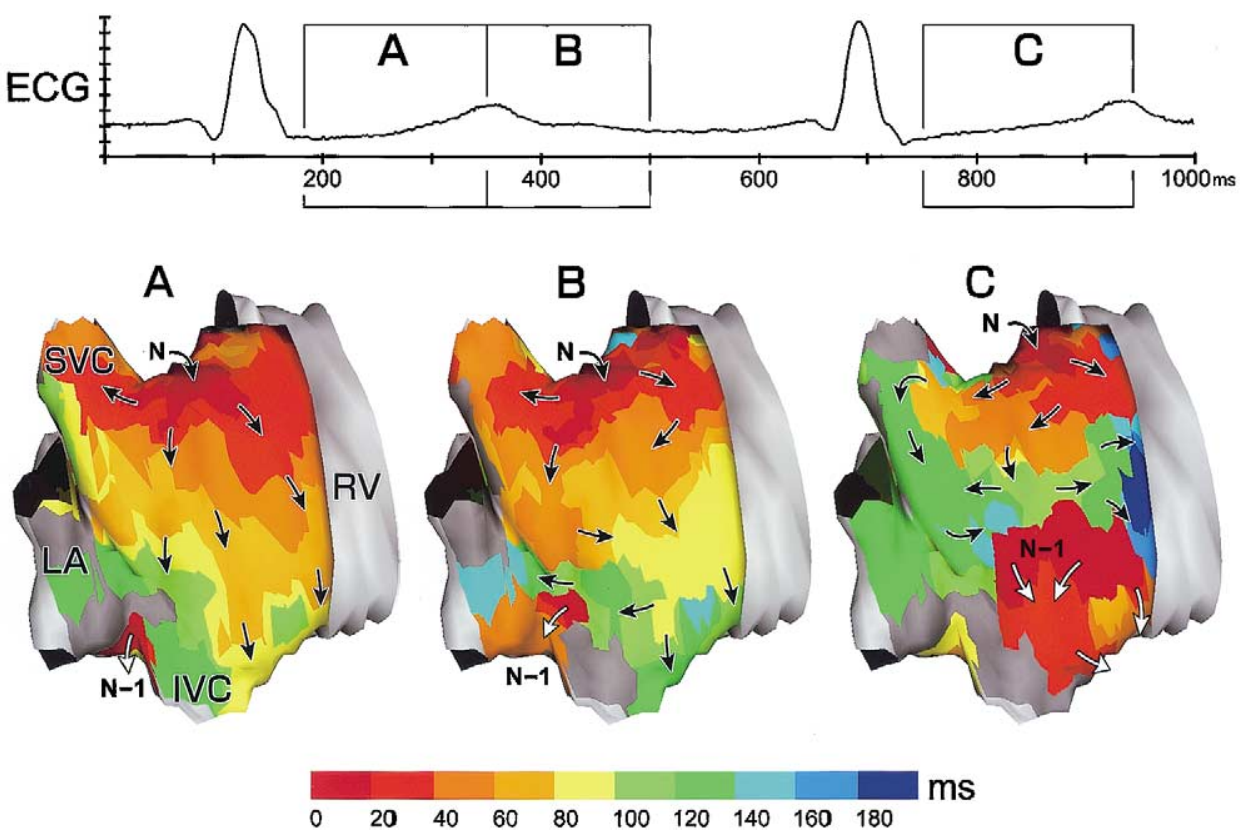

Figure 4. Passive pattern of the right atrial activation demonstrated in a 75-year-old man with severe mitral valve regurgitation, coronary artery disease, and permanent AF. The maps represent the atrial activation in the lateral right atrium during the time windows $A, B$, and $C$, which correspond to the electrocardiogram shown in the upper panel. The right atrial activation is the continuation of the left atrial activation traversing over Bachmann's bundle. The activation reached the right atrial appendage and then propagated inferiorly. The black arrows indicate the activation sequence of the $N$ th cycle and the white arrows indicate the activation sequence of the previous cycle $(N-1)$. Note a progressive slowing of the conduction resulting in residual activation from the preceding cycle and multiple wavelets in the lateral right atrium. $R V$, Right ventricle.

atrium. The activation pattern in the left atrium was also focal in this patient. The location of the focal activation in the right atrium varied from beat to beat and was distributed over the lateral right atrium. There was no geographical correlation to the anatomical structures in the right atrium. Macroreentry circulating around an anatomical obstacle, such as the vena cava or tricuspid valve annulus, was not seen in any patients.

\section{Discussion}

\section{Atrial Activation of Human AF}

There have been few mapping studies performed in patients with AF. Intraoperative studies on the atrial activation of the entire atria ${ }^{4}$ or lateral right atrium ${ }^{5}$ during pacing-induced AF in patients with Wolf-Parkinson-White syndrome demonstrated unstable reentrant circuits of a very short cycle length in the right atrium. Harada and colleagues ${ }^{6}$ mapped the right and left atria separately in patients with mitral valve disease and permanent AF intraoperatively. They observed regular and repetitive activation originating from the left atrium and complex and chaotic activation in the right atrium. From these observations, they proposed that the left atrium acts as an electrical chamber for $\mathrm{AF}$ in patients with mitral valve disease.
In the present study, the entire atrial epicardium was mapped with 253 electrodes simultaneously and the data suggest that concurrent multiple left atrial focal activations combined with fibrillatory conduction and right atrial focal or reentrant activations are the underlying mechanism occurring in coexisting multiple wavelets in patients with permanent $\mathrm{AF}$ associated with mitral valve disease. A progressive conduction delay or block in the pathway from the left atrial focus to the right atrium and in the lateral right atrium causes an irregular and complex right atrial activation that is desynchronized with the left atrium. In addition to the passive activation, focal activation and reentrant activation were also observed in the lateral right atrium. All these complex and desynchronized activations of the right and left atria formed multiple wavelets in the atrial activation and fibrillatory waves in the electrocardiograms (Figure 7).

\section{Focal Activation in the Left Atrium}

Although the electrograms were not recorded directly from the pulmonary veins in the present study, the repetitive activations demonstrated were suggestive of the activation originating from the pulmonary veins. Recent observations during catheter ablation of focal AF have shown that repet- 

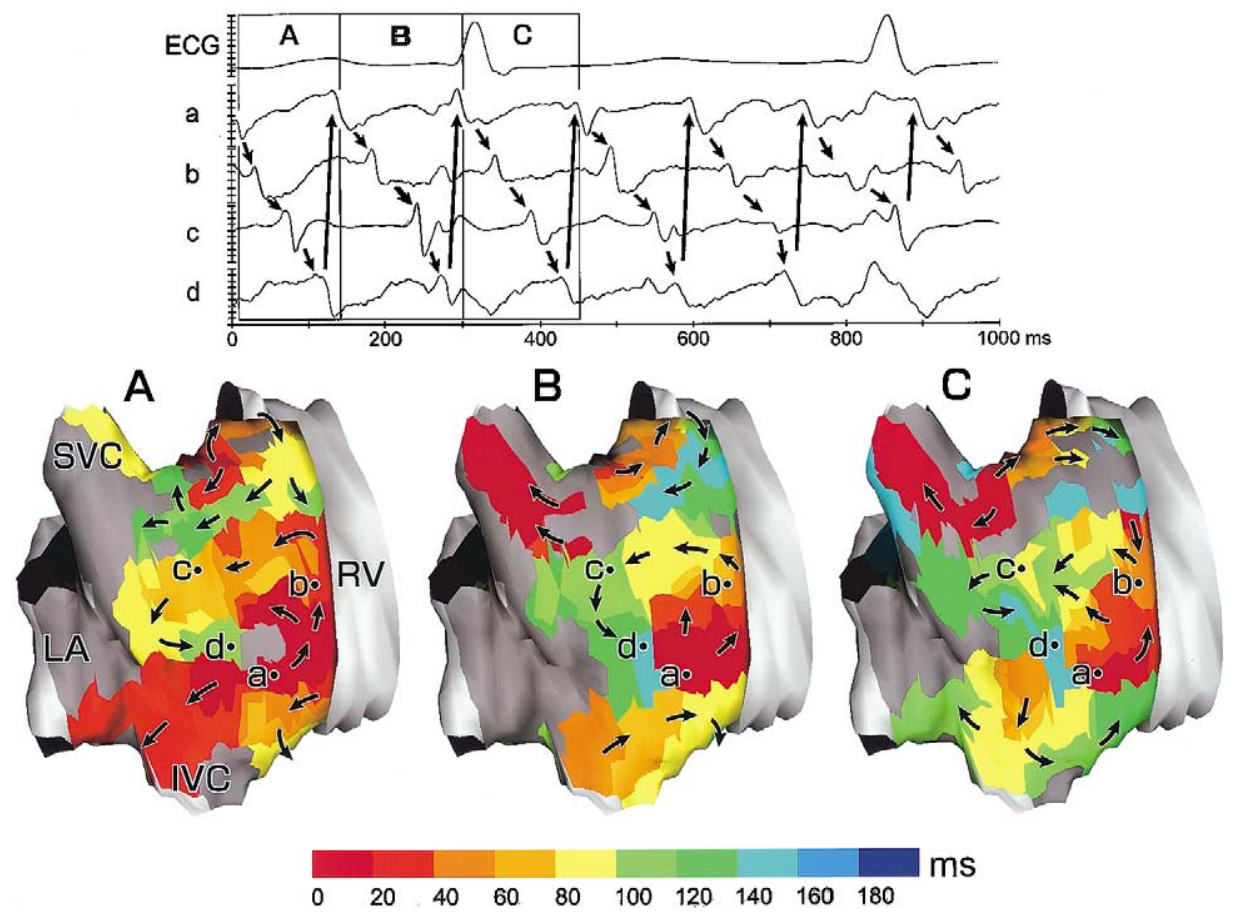

Figure 5. Right atrial activation maps constructed in a 70 -year-old female patient with permanent AF associated with mitral valve stenosis. The maps represent the lateral aspect of the right atrium and are shown along with an electrocardiogram and selected electrograms. The location of those electrodes (a-d) is indicated in the maps. The activation maps during the time windows from $A$ to $C$ are shown. The duration of each time window (the cycle length of the activation recorded from electrode a) is 132, 155, and 155 milliseconds, respectively. The arrows indicate the activation sequence. Note the reentrant activation in the right atrium.
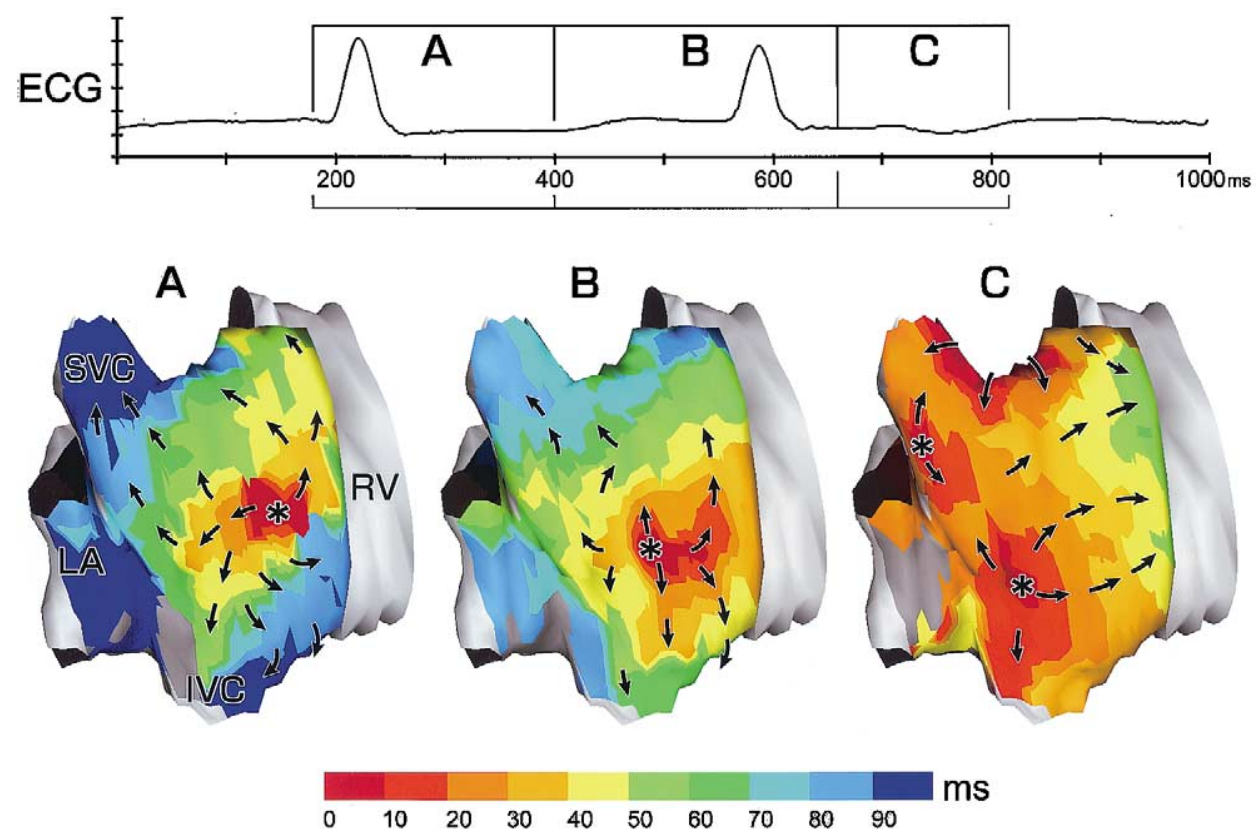

Figure 6. Right atrial activation maps constructed in a 51-year-old female patient with permanent AF associated with aortic valve regurgitation and mitral valve stenosis. Three consecutive right atrial activations are shown. The cycle lengths of these activations are 222, 253, and 162 milliseconds, respectively. Note the focal activation arising in the right atrium. The site of the earliest activation in the right atrium is indicated as an asterisk $(*)$. 


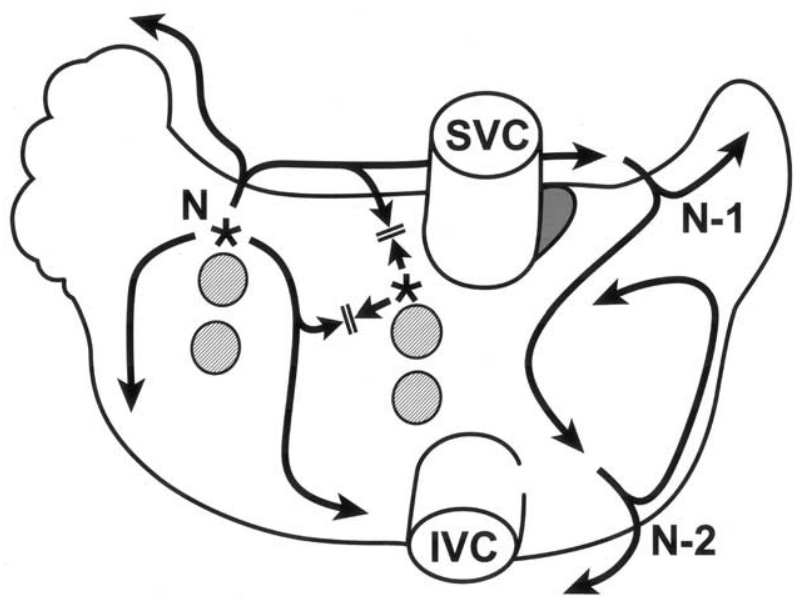

Figure 7. Multiple left atrial focal activations with fibrillatory conduction as a mechanism of permanent AF. The schema represents the posterior aspect of the atria as shown in Figure 3. The asterisks (*) adjacent to the right and left superior pulmonary veins indicate the sites of focal activation. $N-2, N-1$, and $N$ are consecutive activations, arising from the same focus from the left superior pulmonary vein. The activation with a shorter cycle length propagates toward the right atrium, leaving the other activation confined to a limited region. Because of high-frequency focal activations and slow conduction, $N-1$ activation started before $\mathrm{N}-2$ activation had completed an entire atrial activation, and $N$ activation started before $N-1$ activation completed an entire atrial activation.

itive activations arising from the pulmonary veins are the trigger initiating paroxysmal AF. ${ }^{11}$ Studies using magnetic resonance imaging in patients with focal AF have demonstrated that the diameters of the left atrium and the pulmonary veins are significantly larger than in normal subjects. ${ }^{12}$ Because all the patients had a moderate to severe dilated left atrium accompanied by mitral valve disease in the present study, they could have a more severe underlying pathophysiology than that described in patients with focal AF. Stenosis or regurgitation of the mitral valve elevates the pressure and diameter of the pulmonary veins and further provokes the pathophysiological changes underlying the repetitive automatic activation or microreentry in the myocardial sleeves in the veins.

It is interesting that the distribution of the site of focal activation was similar to that of the focal activation reported in paroxysmal AF patients. ${ }^{11}$ The focal activation was frequently observed in the posterior left atrium adjacent to the left and right superior pulmonary veins but less often in the right inferior pulmonary vein. This inhomogeneous distribution of the sources of repetitive focal activation among the pulmonary veins could be explained by a structural difference in the myocardial sleeve between the pulmonary veins. $^{13}$
The present study has shown that the activation was not necessarily regular at the left atrial foci in the permanent $\mathrm{AF}$ patients. One possible explanation for the irregular activation recorded from the posterior left atrium could be that although the pulmonary vein potentials were not directly recorded in this study, a difference in the refractory periods between the pulmonary veins and the left atrium and the decremental conduction in the pulmonary veins ${ }^{14}$ modulated the regular activation in the affected pulmonary vein into the irregular and slower activation in the posterior left atrium. Damaged myocardium resulting from the dilatation of the left atrium could have caused a conduction disturbance between the pulmonary vein and posterior left atrium in the patients with permanent $\mathrm{AF}$.

\section{Fibrillatory Conduction}

Fibrillatory conduction has been shown to produce multiple wavelets in experimental studies. Schuessler and colleagues $^{3}$ demonstrated in an isolated canine right atrial preparation that a single relatively stable high-frequency reentrant circuit provoked by acetylcholine caused multiple wavelets in which, because of the spatial dispersion of the refractoriness and the presence of slow conduction, the propagation wave was blocked or slowed in several areas and resulted in fibrillatory conduction. Mansour's group ${ }^{15}$ showed in their perfused sheep heart preparation that there was a left-to-right gradient in the activation frequency of the atria during high-frequency stimulation of the left atrium and that the increase in the left atrial activation frequency further increased the gradient. Their data suggested that $\mathrm{AF}$ could be the result of high-frequency sources in the left atrium with fibrillatory conduction in the pathway to the right atrium.

\section{Right Atrial Activation}

In addition to the passive activation, focal and reentrant activations were also observed in the right atrium in the present study. Unlike the focal activation in the left atrium, for the foci distributed over the lateral right atrium there was no geographical correlation to the anatomical structures in the right atrium. This is suggestive of a different mechanism or substrate underlying the focal activation in the right atrium compared with that arising from the pulmonary veins. Automaticity provoked by the rapid activation or epicardial breakthrough of the intramural reentry ${ }^{16}$ could be the mechanism for the focal activation in the right atrium.

In the present study, the number of wave fronts was greater in the right atrium than in the left atrium, suggesting that the right atrium had a larger electrophysiological capacity for perpetuation of reentrant activation. Because of the presence of abundant trabeculae, the effective electrophysiological area should be larger in the right atrium than in the left atrium, although the actual anatomical volume 
should be nearly the same. In addition, unique myoarchitecture in the right atrium, such as the crista terminalis and the pectinate muscle arising perpendicularly from it, could further facilitate the perpetuation of reentry.

\section{Rationale for Current Surgical Procedures}

The results of the present study support the concept of the maze procedure ${ }^{17}$ that terminates $\mathrm{AF}$ with a high success rate. The procedure consists of isolation of all pulmonary veins, excision of both atrial appendages, and multiple incisions on both atria. The isolation of the pulmonary veins and excision of the atrial appendages would block propagation of the repetitive activation arising from these regions as shown in the present study. The multiple incisions would block atrial reentry, which was demonstrated as another important mechanism in $\mathrm{AF}$ in the present study. Because all the atrial incisions are extended down to the atrioventricular groove or other anatomical obstacles, all the potential macroreentrant circuits are eliminated.

Recently, simplified surgical procedures confined to the left atrium have been shown to cure AF in selected patients. Sueda and colleagues ${ }^{8}$ performed the left atrial maze procedure, in which the posterior left atrium including all the pulmonary veins were electrically isolated and the atrial incisions were confined to the left atrium, in patients with permanent AF associated with mitral valve disease. They found that approximately $70 \%$ of the patients were cured of $\mathrm{AF}$ without the incisions in the right atrium and the interatrial septum. More recently, they further simplified the procedure to pulmonary vein isolation and obtained a similar result. ${ }^{9}$ The rationale for cure of AF by these simplified procedures might be in blocking propagation of the repetitive activation arising from the pulmonary veins by the isolation of the entire posterior left atrium or the right and left pulmonary veins. The results in the present study suggest that the patients who were not cured of AF by these simplified procedures might have reentry or focal activation in the right atrium and may be cured of AF by the right atrial incisions.

\section{Clinical Implications}

The most exciting implication of the present study would be a map-guided surgery for AF. The present study demonstrated that although the preoperative patient characteristics had no correlation to the atrial activation patterns, intraoperative mapping was able to characterize the atrial activation during $\mathrm{AF}$ and classify the patients into various subtypes, suggesting that all the atrial incisions and cryothermia may not be necessary to cure AF in some patients. If the map-guided surgery is feasible, a more electrophysiologically directed therapeutic strategy would be achieved in the treatment of AF, which would be beneficial in reducing the surgical mortality and morbidity, in- creasing the success rate for $\mathrm{AF}$, and preserving a more atrial mechanical function by avoiding unnecessary incisions. Analysis of the atrial activation during AF would identify patients who may be cured of AF only by the isolation of pulmonary veins, or those who require all the incisions and cryothermia of the maze procedure, or even those who require additional or different procedures to eradicate AF.

In the present study, all the patients had more than 2 foci in the left atrium and the fastest activation propagated toward the right atrium, leaving the other activations confined to a small atrial region. This suggests that ablation of 1 focal site may fail to cure AF because the second focus would assume dominance with surgery for permanent AF. Furthermore, simultaneous mapping of the entire atria would be essential in the map-guided surgery for AF because mapping of the limited atrial region or nonsimultaneous mapping of the separate atrial regions may fail to find the concurrent multiple activations. Even if a patient is cured of AF by the successful ablation of the mapped focal region, atrial mapping and provocation of the focal activation should be repeated to eradicate AF completely.

To achieve successful map-guided surgery for AF, the following issues should be resolved. First, the temporal constancy in the electrophysiological mechanism of AF should be proven in each patient. Because the mechanism of AF can change over time, the mapping should be repeated several times. The effect of anesthesia on the atrial electrophysiology should also be considered. Second, the duration of the mapping and analysis of the data should be shortened. Although our 256-channel 3-dimensional mapping system is capable of analyzing complex and concurrent activations during $\mathrm{AF}$ and displaying multiple wave fronts in a dynamic mode as a movie on 3-dimensionally constructed atrial models, it still requires a considerable time for analysis of the complex atrial electrograms during AF. Last, an offpump surgical technique that creates linear atrial incisions and annular cryothermia without a need for cardiopulmonary bypass and cardioplegic arrest should be established. The technique not only reduces the complications related to the cardiopulmonary bypass and cardioplegic arrest but also enables real-time verification of conduction block across each incision and further facilitates electrophysiological assessment of AF. Recently, microwave energy ${ }^{18}$ or the bipolar application of radiofrequency energy ${ }^{19}$ have been shown to produce transmural linear lesions on the beating heart.

\section{Study Limitations}

All the patients had been in AF for 3 years or more in the present study. The short duration of 4 seconds of data might not have been enough to determine the atrial activation during $\mathrm{AF}$ in these patients. As mentioned above, repeated 
data acquisition in a different instance may be necessary to examine whether there would be a time variation in the atrial activation pattern and characteristics demonstrated in the present study. Further, the data were recorded during surgery under general anesthesia. The anesthetic drugs might affect the autonomic nerve system or directly change the atrial electrophysiology.

This was an epicardial mapping study and the electrograms were not recorded from the interatrial septum or coronary sinus. Because macroreentry circulating in the lateral right atrium and interatrial septum was frequently observed in the canine pericardial model of $\mathrm{AF}^{20}$ macroreentry conducting through the interatrial septum could have participated in the perpetuation of $\mathrm{AF}$ in our patients also. Further, the interatrial electrical connections through the coronary sinus musculature or the interatrial septum might have acted as pathways for interatrial conduction during $\mathrm{AF}$, and the focal activation occasionally observed in the lateral left atrium could have been a breakthrough of the right-to-left activation propagating through the coronary sinus musculature. Preoperative biatrial endocardial mapping using a basket catheter or noncontact balloon catheter would solve these problems and provide additional information on the mechanism of AF.

We acknowledge the excellent technical assistance of Motoaki Uchiyama. We also thank Hitomi Horiguchi for preparation of the illustrations and John Martin for preparation of the manuscript.

\section{References}

1. Moe GK. On the multiple wavelet hypothesis of atrial fibrillation. Arch Int Pharmacodyn Ther. 1962;140:183-8.

2. Allessie MA, Bonke FI, Schopman FJ. Circus movement in rabbit atrial muscle as a mechanism of tachycardia. Circ Res. 1973;33:54-62.

3. Schuessler RB, Grayson TM, Bromberg BI, Cox JL, Boineau JP. Cholinergically mediated tachyarrhythmias induced by a single extrastimulus in the isolated canine right atrium. Circ Res. 1992;71:125467.

4. Cox JL, Canavan TE, Schuessler RB, et al. The surgical treatment of atrial fibrillation. II. Intraoperative electrophysiologic mapping and description of the electrophysiologic basis of atrial flutter and atrial fibrillation. J Thorac Cardiovasc Surg. 1991;101:406-26.

5. Konings KT, Kirchhof CJ, Smeets JR, Wellens HJ, Penn OC, Allessie MA. High-density mapping of electrically induced atrial fibrillation in humans. Circulation. 1994;89:1665-80.

6. Harada A, Sasaki K, Fukushima T, et al. Atrial activation during chronic atrial fibrillation in patients with isolated mitral valve disease. Ann Thorac Surg. 1996;61:104-11.

7. Yamauchi S, Ogasawara H, Saji Y, Bessho R, Miyagi Y, Fujii M. Efficacy of intraoperative mapping to optimize the surgical ablation of atrial fibrillation in cardiac surgery. Ann Thorac Surg. 2002;74:450-7.

8. Sueda T, Nagata H, Shikata $H$, et al. Simple left atrial procedure for chronic atrial fibrillation associated with mitral valve disease. Ann Thorac Surg. 1996;62:1796-800.

9. Sueda T, Imai K, Ishii O, Orihashi K, Watari M, Okada K. Efficacy of pulmonary vein isolation for the elimination of chronic atrial fibrillation in cardiac valvular surgery. Ann Thorac Surg. 2001;71:1189-93.

10. Nitta T, Sakamoto S, Miyagi Y, et al. Initial experience with the radial incision approach for atrial fibrillation. Ann Thorac Surg. 1999;68: 805-11.

11. Haissaguerre M, Jais P, Shah DC, et al. Spontaneous initiation of atrial fibrillation by ectopic beats originating in the pulmonary veins. $N$ Engl J Med. 1998;339:659-66.

12. Tsao HM, Yu WC, Cheng $\mathrm{HC}$, et al. Pulmonary vein dilation in patients with atrial fibrillation: detection by magnetic resonance imaging. J Cardiovasc Electrophysiol. 2001;12:809-13.

13. Nathan H, Eliakim M. The junction between the left atrium and the pulmonary veins. An anatomic study of human hearts. Circulation. 1966;34:412-22.

14. Jaïs P, Hocini M, Macle L, et al. Distinctive electrophysiological properties of pulmonary veins in patients with atrial fibrillation. Circulation. 2002;106:2479-85.

15. Mansour M, Mandapati R, Berenfeld O, Chen J, Samie FH, Jalife J. Left-to-right gradient of atrial frequencies during acute atrial fibrillation in the isolated sheep heart. Circulation. 2001;103:2631-6.

16. Schuessler RB, Kawamoto T, Hand DE, et al. Simultaneous epicardial and endocardial activation sequence mapping in the isolated canine right atrium. Circulation. 1993;88:250-63.

17. Cox JL, Schuessler RB, D’Agostino HJ Jr, et al. The surgical treatment of atrial fibrillation. III. Development of a definitive surgical procedure. J Thorac Cardiovasc Surg. 1991;101:569-83.

18. Maessen JG, Nijs JF, Smeets JL, Vainer J, Mochtar B. Beating-heart surgical treatment of atrial fibrillation with microwave ablation. Ann Thorac Surg. 2002;74:1307-11.

19. Prasad SM, Maniar HS, Schuessler RB, Damiano RJ Jr. Chronic transmural atrial ablation by using bipolar radiofrequency energy on the beating heart. $J$ Thorac Cardiovasc Surg. 2002;124:708-13.

20. Kumagai K, Khrestian C, Waldo AL. Simultaneous multisite mapping studies during induced atrial fibrillation in the sterile pericarditis model. Insights into the mechanism of its maintenance. Circulation. 1997;95:511-21. 